Article

\title{
Mathematical Aspects of Krätzel Integral and Krätzel Transform
}

\author{
Arak M. Mathai ${ }^{1}$ and Hans J. Haubold ${ }^{2, *(1)}$ \\ 1 Department of Mathematics and Statistics, McGill University, Montreal, PQ H3A 2K6, Canada; \\ a.mathai@mcgill.ca \\ 2 Office for Outer Space Affairs, United Nations, Vienna International Centre, A-1400 Vienna, Austria \\ * Correspondence: hans.haubold@gmail.com
}

Received: 11 February 2020; Accepted: 22 March 2020; Published: 3 April 2020

\begin{abstract}
A real scalar variable integral is known in the literature by different names in different disciplines. It is basically a Bessel integral called specifically Krätzel integral. An integral transform with this Krätzel function as kernel is known as Krätzel transform. This article examines some mathematical properties of Krätzel integral, its connection to Mellin convolutions and statistical distributions, its computable representations, and its extensions to multivariate and matrix-variate cases, in both the real and complex domains. An extension in the pathway family of functions is also explored.
\end{abstract}

Keywords: Mellin convolutions; Krätzel integrals; reaction-rate probability integral; continuous mixtures; Bayesian structures; fractional integrals; statistical distribution of products and ratios; multivariate and matrix-variate cases; real and complex domains

MSC: 26B12; 26A33; 60E10; 62E15; 33C60

\section{Introduction}

In this paper, real scalar mathematical or random variables are denoted by small letters $x, y, z, \ldots$ and the corresponding vector/matrix variables are denoted by capital letters $X, Y, \ldots$ Variables in the complex domain are denoted with a tilde such as $\tilde{x}, \tilde{y}, \tilde{X}, \tilde{Y} \ldots .$. Constant vectors /matrices are denoted by capital letters $A, B, \ldots$ whether in the real or complex domain. Scalar constants are denoted by $a, b, \ldots$. If $X=\left(x_{i j}\right)$ is a $p \times q$ matrix where the $x_{i j}$ s are distinct real scalar variables, then the wedge product of the differentials is denoted by $\mathrm{d} X=\wedge_{i=1}^{p} \wedge_{j=1}^{q} \mathrm{~d} x_{i j}$. If $x$ and $y$ are real scalar variables, then the wedge product of their differentials is defined as $\mathrm{d} x \wedge \mathrm{d} y=-\mathrm{d} y \wedge \mathrm{d} x$ so that $\mathrm{d} x \wedge \mathrm{d} x=0, \mathrm{~d} y \wedge \mathrm{d} y=0$. If $\tilde{X}$ is in the complex domain, then $\tilde{X}=X_{1}+i X_{2}$ where $X_{1}, X_{2}$ are real and $i=\sqrt{(-1)}$. Then, $\mathrm{d} \tilde{X}=\mathrm{d} X_{1} \wedge \mathrm{d} X_{2}$. The determinant of a $p \times p$ real matrix $X$ is denoted by $|X| \operatorname{or} \operatorname{det}(X)$ and when in the complex domain the absolute value of the determinant is denoted by $|\operatorname{det}(X)|$. The trace of a square matrix $A$ is denoted by $\operatorname{tr}(A)$. The integral

$$
\int_{A}^{B} f(X) \mathrm{d} X=\int_{O<A<X<B} f(X) \mathrm{d} X
$$

means a real-valued scalar function $f(X)$ of the $p \times p$ real positive definite matrix $X$ is integrated out over $X>O$ (positive definite), $X-A>O, B-X>O, A>O, B>O$ where $A$ and $B$ are $p \times p$ constant positive definite matrices. The corresponding integral in the complex domain is denoted as $\int_{A}^{B} \tilde{f}(\tilde{X}) \mathrm{d} \tilde{X}$. 


\subsection{Krätzel Integral}

Let $x$ be a real scalar variable. Consider the following integrals:

$$
\begin{gathered}
K_{1}=\int_{0}^{\infty} x^{\gamma-1} \mathrm{e}^{-a x-\frac{b}{x}} \mathrm{~d} x, a>0, b>0, \gamma>0 \\
K_{2}=\int_{0}^{\infty} x^{\gamma-1} \mathrm{e}^{-a x^{\delta}-b x^{-\rho}} \mathrm{d} x, a>0, b>0, \gamma>0, \delta>0, \rho>0 .
\end{gathered}
$$

This $K_{2}$ in Equation (2) is known as the generalized Krätzel integral and Equation (1) as the basic Krätzel integral. When $\delta=1$ in Equation (2), we have the Laplace transform of $x^{\gamma-1} \mathrm{e}^{-b x^{-\rho}}$ with Laplace parameter $a$. For $\delta=1, \rho=\frac{1}{2}$ in Equation (2), we have the basic reaction-rate probability integral in nuclear and solar neutrino astrophysics (see [1,2]). When $\delta=1, \rho=1$, the integrand in Equation (1) is the inverse Gaussian density for appropriate values of $a, b, \gamma$ and multiplied by a normalizing constant. In addition, Equation (2) is a generalized situation of the same and Equation (1) provides the moment expression for the inverse Gaussian density, multiplied by a normalizing constant. Krätzel transform is associated with Equation (1) (see [3]). Some authors call Equation (2) as the generalized gamma, ultra gamma, Bessel integral, etc. In [4], it is shown that in the simple poles case it is a Bessel series and hence it is more appropriate to call it as a generalized Bessel integral.

The highlight of the present discussion is to point out the importance and usefulness of Krätzel function in various topics in widely different areas and to consider its extensions of various types. Krätzel integrals appear in Mellin convolution of product of two functions; in statistical distribution theory as the density of a product of two independently distributed generalized gamma random variables; in Bayesian analysis when the conditional and marginal densities belong to generalized gamma densities; in model building, especially in the pathway models where the limiting forms end up in Krätzel functions; in nuclear reaction-rate theory; and in inverse Gaussian models in stochastic processes, to mention a few topics. Krätzel function is also associated with generalized gamma and ultra gamma integrals, Kobayashi integrals and generalized special functions such as G- and $\mathrm{H}$-functions. In the present discussion, we also consider extensions of Krätzel function to multivariate cases involving many scalar variables, matrix-variate cases in the real and complex domains and extensions involving multiple integrals.

\subsection{Evaluation of the Integral in Equation (2)}

One can evaluate Equation (2) by using different approaches. One can interpret Equation (2) as the Mellin convolution of a product and then take the inverse Mellin transform to evaluate the integral. One can draw a parallel to the statistical density of a product of two positive real scalar random variables and then evaluate the density to obtain the value of Equation (2). One can treat Equation (2) as a function $g(b)$ of $b$. Then, the Mellin transform of $g(b)$ with Mellin parameter $s$ is the following for $\gamma>0, \delta>0, a>0, b>0, \eta>0$ :

$$
\begin{aligned}
M_{g}(s) & =\int_{0}^{\infty} b^{s-1}\left\{\int_{0}^{\infty} x^{\gamma-1} \mathrm{e}^{-a x^{\delta}-b x^{-\rho}} \mathrm{d} x\right\} \mathrm{d} b \\
& =\int_{0}^{\infty} \int_{0}^{\infty} b^{s-1} x^{\gamma-1} \mathrm{e}^{-a x^{\delta}-b x^{-\rho}} \mathrm{d} x \wedge \mathrm{d} b .
\end{aligned}
$$

Integrating out $b$ first and then $x$, we have the following:

$$
\begin{aligned}
\int_{0}^{\infty} b^{s-1} \mathrm{e}^{-b x^{-\rho}} \mathrm{d} b & =\Gamma(s) x^{\rho s}, \Re(s)>0 \\
\int_{0}^{\infty} x^{\gamma+\rho s-1} \mathrm{e}^{-a x^{\delta}} \mathrm{d} x & =\frac{1}{\delta} \Gamma\left(\frac{\gamma+\rho s}{\delta}\right) a^{-\left(\frac{\gamma+\rho s}{\delta}\right)}, \Re(\gamma+\rho s)>0
\end{aligned}
$$


where $\Re(\cdot)$ means the real part of $(\cdot)$. That is,

$$
M_{g}(s)=\frac{1}{\delta a^{\frac{\gamma}{\delta}}} \Gamma(s) \Gamma\left(\frac{\gamma+\rho s}{\delta}\right) a^{-\frac{\rho}{\delta} s} .
$$

Taking the inverse Mellin transform of Equation (3) we have $g(b)$ or the integral in Equation (2) as the following:

$$
K_{2}=\frac{1}{\delta a^{\frac{\gamma}{\delta}}} \frac{1}{2 \pi i} \int_{c-i \infty}^{c+i \infty} \Gamma(s) \Gamma\left(\frac{\gamma}{\delta}+\frac{\rho}{\delta} s\right)\left(b a^{\frac{\rho}{\delta}}\right)^{-s} \mathrm{~d} s, i=\sqrt{(-1)}
$$

where the $c$ in the contour is $>0$. Note that Equation (4) can be written as a H-function.

$$
K_{2}=\frac{1}{\delta a^{\frac{\gamma}{\delta}}} H_{0,2}^{2,0}\left[\left.b a^{\frac{\rho}{\delta}}\right|_{(0,1),\left(\frac{\gamma}{\delta}, \frac{\rho}{\delta}\right)}\right] .
$$

For the theory and applications of the H-function, see [5]. When $\rho=\delta$, we have Equation (5) reducing to a Meijer's G-function as the following:

$$
K_{2}=\frac{1}{\delta a^{\frac{\gamma}{\delta}}} G_{0,2}^{2,0}\left[\left.a b\right|_{0, \frac{\gamma}{\delta}}\right] .
$$

For the theory and applications of G-function, see [6].

\subsection{Computable Series form for Equation (2)}

Consider the Mellin-Barnes integral representation in Equation (4). This integral can be evaluated as the sum of the residues at the poles of the gammas $\Gamma(s)$ and $\Gamma\left(\frac{\gamma}{\delta}+\frac{\rho}{\delta} s\right)$. The poles of $\Gamma(s)$ are at $s=0,-1,-2, \ldots$. When the poles of the integrand are simple. then the sum of the residues at the poles of $\Gamma(s)$ is the following:

$$
\text { (A) } \left.\delta a^{\frac{\gamma}{\delta}}\right)^{-1} \sum_{v=0}^{\infty} \frac{(-1)^{v}}{v !} \Gamma\left(\frac{\gamma}{\delta}-\frac{\rho}{\delta} v\right)\left(b a^{\frac{\rho}{\delta}}\right)^{v} .
$$

The poles of $\Gamma\left(\frac{\gamma}{\delta}+\frac{\rho}{\delta} s\right)$ are at $\frac{\gamma}{\delta}+\frac{\rho}{\delta} s=-v, v=0,1,2, \ldots$ or the poles are at $s=-\frac{\gamma}{\rho}-\frac{\delta}{\rho} v$ and in the simple poles case the sum of the residues is the following:

$$
\text { (B) } \frac{b^{\frac{\gamma}{\rho}}}{\delta} \sum_{\nu=0}^{\infty} \frac{(-1)^{v}}{v !} \Gamma\left(-\frac{\gamma}{\rho}-\frac{\delta}{\rho} v\right)\left(a b^{\frac{\delta}{\rho}}\right)^{\nu} .
$$

Hence, the sum of residues from $(A)$ and $(B)$ in the simple poles case is the following:

$$
\begin{aligned}
K_{2} & =\left(\delta a^{\frac{\gamma}{\delta}}\right)^{-1} \sum_{v=0}^{\infty} \frac{(-1)^{v}}{v !} \Gamma\left(\frac{\gamma}{\delta}-\frac{\rho}{\delta} v\right)\left(b a^{\frac{\rho}{\delta}}\right)^{v} \\
& +\frac{b^{\frac{\gamma}{\rho}}}{\delta} \sum_{v=0}^{\infty} \frac{(-1)^{v}}{v !} \Gamma\left(-\frac{\gamma}{\rho}-\frac{\delta}{\rho} v\right)\left(a b^{\frac{\delta}{\rho}}\right)^{v} .
\end{aligned}
$$

\subsection{G-function in the Simple Poles Case}

Let $\rho=\delta$ so that the H-function in Equation (5) becomes the G-function in Equation (6) and when $\frac{\gamma}{\delta}$ is not an integer then the G-function has simple poles. Consider this case and it is available from Equation (7) by putting $\delta=\rho$. Then, the gammas reduce to the following:

$$
\Gamma\left(\frac{\gamma}{\rho}-v\right)=\frac{\Gamma\left(\frac{\gamma}{\rho}\right)}{(-1)^{v}\left(-\frac{\gamma}{\rho}+1\right)_{v}} \text { and } \Gamma\left(-\frac{\gamma}{\rho}-v\right)=\frac{\Gamma\left(-\frac{\gamma}{\rho}\right)}{(-1)^{v}\left(\frac{\gamma}{\rho}+1\right)_{v}}
$$


where, in general, the notation $(a)_{m}=a(a+1) \ldots(a+m-1), a \neq 0,(a)_{0}=1$ is the Pochhammer symbol. Hence, $K_{2}$ in Equation (2) for this simple poles case and for $\delta=\rho$ is the following:

$$
\begin{aligned}
K_{2} & =\frac{\Gamma\left(\frac{\gamma}{\rho}\right)}{\rho a^{\frac{\gamma}{\rho}}} \sum_{\nu=0}^{\infty} \frac{1}{\left(-\frac{\gamma}{\rho}+1\right)_{v} v !}(a b)^{v}+\frac{\Gamma\left(-\frac{\gamma}{\rho}\right) b^{\frac{\gamma}{\rho}}}{\rho} \sum_{\nu=0}^{\infty} \frac{1}{\left(\frac{\gamma}{\rho}+1\right)_{\nu} v !}(a b)^{v} \\
& =\frac{\Gamma\left(\frac{\gamma}{\rho}\right)}{\rho a^{\frac{\gamma}{\rho}}}{ }_{0} F_{1}\left(;-\frac{\gamma}{\rho}+1 ; a b\right)+\frac{\Gamma\left(-\frac{\gamma}{\rho}\right) b^{\frac{\gamma}{\rho}}}{\rho}{ }_{0} F_{1}\left(; \frac{\gamma}{\rho}+1 ; a b\right),
\end{aligned}
$$

where ${ }_{0} F_{1}$ is a hypergeometric series with no upper and one lower parameters. Observe that, in this simple poles case, Equation (2) or $K_{2}$ of Equation (8) is a linear function of Bessel series and hence it is appropriate to call Equation (1) as Bessel integral and Equation (2) as the generalized Bessel integral rather than calling them as ultra gamma integral or generalized gamma integral or anything connected with gamma integral.

\subsection{Poles of Order Two, $\rho=\delta, \frac{\gamma}{\delta}=m, m=1,2, \ldots$}

In this case, the poles at $s=0,-1,-2, \ldots,-(m-1)$ are simple and poles at $s=-m,-m-1, \ldots$ are of order two each. In this case, we may write (2) as the following:

$$
K_{2}=\frac{1}{\rho a^{\frac{\gamma}{\rho}}} \frac{1}{2 \pi i} \int_{c-i \infty}^{c+i \infty} \Gamma(s) \Gamma(m+s)(a b)^{-s} \mathrm{~d} s .
$$

Sum of the residues at the poles $s=0,-1, \ldots-(m-1)$, coming from (9), is the following:

$$
\text { (C) } \frac{1}{\rho a^{\frac{\gamma}{\rho}}} \sum_{v=0}^{m-1} \frac{(-1)^{v}}{v !} \Gamma(m-v)(a b)^{v} \text {. }
$$

For $s=-m-v, v=0,1, \ldots$ or $s=-v, v=m, m+1, \ldots$ the poles are of order two and the residue, denoted by $R_{v}$, is the following: Let $h(s)=\Gamma(s) \Gamma(m+s)(a b)^{-s}$. Then,

$$
\begin{aligned}
R_{v} & =\lim _{s \rightarrow-v} \frac{\mathrm{d}}{\mathrm{d} s}\left[(s+v)^{2} \Gamma(s) \Gamma(m+s)(a b)^{-s}\right] \\
& =\lim _{s \rightarrow-v} \frac{\mathrm{d}}{\mathrm{d} s}\left[(s+v)^{2} \frac{(s+v-1)^{2} \ldots(s+m)^{2}}{(s+v-1)^{2} \ldots(s+m)^{2}} \frac{(s+m-1) \ldots s}{(s+m-1) \ldots s} \Gamma(s) \Gamma(m+s)(a b)^{-s}\right] \\
& =\lim _{s \rightarrow-v} \frac{\mathrm{d}}{\mathrm{d} s}\left[\frac{\Gamma^{2}(s+v+1)}{(s+v-1)^{2} \ldots(s+m)^{2}(s+m-1) \ldots s}(a b)^{-s}\right] .
\end{aligned}
$$

Observe that $\frac{\mathrm{d}}{\mathrm{d} s} h(s)=h(s) \frac{\mathrm{d}}{\mathrm{d} s} \ln h(s)$ and $(a b)^{-s}=\mathrm{e}^{-s \ln (a b)}$. Note that

$$
\begin{aligned}
\lim _{s \rightarrow-v} h(s) & =\frac{(-1)^{m}(a b)^{v}}{v !(v-m) !}, v=m, m+1, \ldots \\
\lim _{s \rightarrow-v} \frac{\mathrm{d}}{\mathrm{d} s} \ln h(s) & =\lim _{s \rightarrow-v}\left[2 \psi(s+v+1)-\frac{2}{s+v-1}-\ldots-\frac{2}{s+m}\right. \\
& \left.-\frac{1}{s+m-1}-\ldots-\frac{1}{s}-\ln (a b)\right] \\
& =2 \psi(1)+2\left[1+\frac{1}{2}+\ldots+\frac{1}{v-m}\right]+\left[\frac{1}{v-m+1}+\ldots+\frac{1}{v}\right]-\ln (a b) \\
& =\psi(v+1)+\psi(v-m+1)-\ln (a b) .
\end{aligned}
$$


Therefore,

$$
R_{v}=[\psi(v+1)+\psi(v-m+1)-\ln (a b)]\left[\frac{(-1)^{m}(a b)^{v}}{v !(v-m) !}\right], v=m, m+1, \ldots .
$$

Then, in this case, (2) reduces to the following:

$$
\begin{aligned}
K_{2} & =\frac{1}{\rho a^{\frac{\gamma}{\rho}}} \sum_{v=0}^{m-1} \frac{(-1)^{v}}{v !} \Gamma(m-v)(a b)^{v} \\
& +\sum_{v=m}^{\infty}[\psi(v+1)+\psi(v-m+1)-\ln (a b)]\left[\frac{(-1)^{m}}{v !(v-m) !}(a b)^{v}\right], v=m, m+1, \ldots
\end{aligned}
$$

where $\psi(\cdot)$ is the psi function or the logarithmic derivative of the gamma function, $\psi(z)=\frac{\mathrm{d}}{\mathrm{d} z} \ln \Gamma(z)$.

The most general case is to consider $\Gamma(s) \Gamma\left(\frac{\gamma}{\delta}+\frac{\rho}{\delta} s\right)$ having some poles of order one and the remaining of order two. After writing this situation in a convenient way, one can use the procedure in Section 1.5 to obtain the final result. Since the expressions would take up too much space, it is not discussed here.

\section{Krätzel Integral from Mellin Convolution}

Let $x_{1}>0$ and $x_{2}>0$ be real scalar variables. Let $f_{1}\left(x_{1}\right)$ and $f_{2}\left(x_{2}\right)$ be real-valued scalar functions associated with $x_{1}$ and $x_{2}$, respectively. Then, the Mellin transforms of $f_{1}$ and $f_{2}$, with Mellin parameter $s$, are the following, whenever they exist:

$$
M_{f_{1}}(s)=\int_{0}^{\infty} x_{1}^{s-1} f_{1}\left(x_{1}\right) \mathrm{d} x_{1}, M_{f_{2}}(s)=\int_{0}^{\infty} x_{2}^{s-1} f_{2}\left(x_{2}\right) \mathrm{d} x_{2} .
$$

Then,

$$
\begin{aligned}
M_{f_{1}}(s) M_{f_{2}}(s) & =\int_{0}^{\infty} \int_{0}^{\infty} x_{1}^{s-1} x_{2}^{s-1} f_{1}\left(x_{1}\right) f_{2}\left(x_{2}\right) \mathrm{d} x_{1} \wedge \mathrm{d} x_{2} \\
& =\int_{0}^{\infty} \int_{0}^{\infty} u^{s-1} f_{1}(v) f_{2}\left(\frac{u}{v}\right) \frac{1}{v} \mathrm{~d} u \wedge \mathrm{d} v, u=x_{1} x_{1}, v=x_{1} \\
& =\int_{0}^{\infty} u^{s-1} g(u) \mathrm{d} u
\end{aligned}
$$

where

$$
\begin{aligned}
g(u) & =\int_{0}^{\infty} \frac{1}{v} f_{1}(v) f_{2}\left(\frac{u}{v}\right) \mathrm{d} v \\
& =\int_{0}^{\infty} \frac{1}{v} f_{1}\left(\frac{u}{v}\right) f_{2}(v) \mathrm{d} v
\end{aligned}
$$

That is,

$$
M_{g}(s)=M_{f_{1}}(s) M_{f_{2}}(s) .
$$

This Equation (12) is the Mellin convolution of the product involving two functions and Equation (11) is the corresponding integral representation. Let $f_{1}$ and $f_{2}$ be generalized exponential functions of the following types:

$$
\text { (D) } f_{j}\left(x_{j}\right)=x_{j}^{\gamma_{j}-1} \mathrm{e}^{-a_{j} x_{j} \delta_{j}}, a_{j}>0, \delta_{j}>0, \gamma_{j}>0, j=1,2
$$


Then, Equation (11) becomes the following:

$$
\text { (E) } \begin{aligned}
g(u) & =u^{\gamma_{2}-1} \int_{0}^{\infty} v^{\gamma_{1}-\gamma_{2}-1} \mathrm{e}^{-a_{1} v^{\delta_{1}}-a_{2}\left(\frac{u}{v}\right)^{\delta_{2}}} \mathrm{~d} v \\
(F) & =u^{\gamma_{1}-1} \int_{0}^{\infty} v^{\gamma_{2}-\gamma_{1}-1} \mathrm{e}^{-a_{1}\left(\frac{u}{v}\right)^{\delta_{1}}-a_{2} v^{\delta_{2}}} \mathrm{~d} v
\end{aligned}
$$

Here, (E) and (Fi) provide equivalent representations for $g(u)$. In $(E)$, if $\delta_{1}=\delta, a_{1}=a, \delta_{2}=\rho, a_{2} u^{\delta_{2}}=$ $b, \gamma_{1}-\gamma_{2}=\gamma$, then the integral becomes Krätzel integral of (2) in Section 1. Hence, Krätzel integral is also available as a Mellin convolution of a product involving two functions, see [7].

Instead of taking $f_{j}\left(x_{j}\right)$ of the form in $(D)$, if we take $f_{1}\left(x_{1}\right)=\frac{1}{\Gamma(\alpha)} x_{1}^{\gamma}\left(1-x_{1}\right)^{\alpha-1}$ for $\Re(\gamma)>$ $-1, \Re(\alpha)>0$ or $\alpha>0, \gamma>-1$ when real, and $f_{2}\left(x_{2}\right)=f\left(x_{2}\right)$ where $f\left(x_{2}\right)$ is an arbitrary function, then Equation (11) becomes the following:

$$
\begin{aligned}
g(u) & =\int_{v} \frac{1}{v} f_{1}\left(\frac{u}{v}\right) f_{2}(v) \mathrm{d} v=\int_{v} \frac{1}{\Gamma(\alpha)} \frac{1}{v}\left(\frac{u}{v}\right)^{\gamma}\left(1-\frac{u}{v}\right)^{\alpha-1} f(v) \mathrm{d} v, \Re(\alpha)>0, \Re(\gamma)>-1 \\
& =\frac{u^{\gamma}}{\Gamma(\alpha)} \int_{v \geq u} v^{-\gamma-\alpha}(v-u)^{\alpha-1} f(v) \mathrm{d} v=K_{2, \gamma}^{-\alpha} f
\end{aligned}
$$

where $K_{2, \gamma}^{-\alpha} f$ in (13) is Erdélyi-Kober fractional integral of the second kind of order $\alpha$ and parameter $\gamma$, see [8]. Thus, the Mellin convolution of a product is also associated with fractional integral of the second kind. A general definition of all versions of fractional integrals in terms of Mellin convolutions of products and ratios is given in [8].

\section{Krätzel Integral as the Density of a Product}

Let $x_{1}>0$ and $x_{2}>0$ be two real scalar positive random variables, independently distributed with density functions $f_{1}\left(x_{1}\right)$ and $f_{2}\left(x_{2}\right)$, respectively. Due to statistical independence their joint density, denoted by $f\left(x_{1}, x_{2}\right)$, is the product, $f\left(x_{1}, x_{2}\right)=f_{1}\left(x_{1}\right) f_{2}\left(x_{2}\right)$. Let $u=x_{1} x_{2}$ be the product and let $x_{1}=v$ or $x_{2}=v$. Then, $\mathrm{d} x_{1} \wedge \mathrm{d} x_{2}=\frac{1}{v} \mathrm{~d} u \wedge \mathrm{d} v$. Let $g(u, v)$ be the joint density of $u$ and $v$. Then,

$$
g(u, v)=\frac{1}{v} f_{1}(v) f_{2}\left(\frac{u}{v}\right)=\frac{1}{v} f_{1}\left(\frac{u}{v}\right) f_{2}(v)
$$

and the marginal density of $u$, denoted by $g_{1}(u)$ is the following:

$$
\begin{aligned}
g_{1}(u) & =\int_{v} \frac{1}{v} f_{1}(v) f_{2}\left(\frac{u}{v}\right) \mathrm{d} v \\
& =\int_{v} \frac{1}{v} f_{1}\left(\frac{u}{v}\right) f_{2}(v) \mathrm{d} v .
\end{aligned}
$$

Let $f_{j}\left(x_{j}\right)$ be a generalized gamma density of the form

$$
f_{j}\left(x_{j}\right)=c_{j} x_{j}^{\gamma_{j}-1} \mathrm{e}^{-a_{j} x_{j} x_{j}}, a_{j}>0, \gamma_{j}>0, \delta_{j}>0, j=1,2
$$

where $c_{j}$ is the normalizing constant. For the $f_{j}\left(x_{j}\right)$ in Equation (15), we have Equation (14) as the following:

$$
\begin{aligned}
g_{1}(u) & =c_{1} c_{2} u^{\gamma_{2}-1} \int_{0}^{\infty} v^{\gamma_{1}-\gamma_{2}-1} \mathrm{e}^{-a_{1} v^{\delta_{1}}-a_{2}\left(\frac{u}{v}\right)^{\delta_{2}}} \mathrm{~d} v \\
& =c_{1} c_{2} u^{\gamma_{1}-1} \int_{0}^{\infty} v^{\gamma_{2}-\gamma_{1}-1} \mathrm{e}^{-a_{1}\left(\frac{u}{v}\right)^{\delta_{1}}-a_{2} v^{\delta_{2}}} \mathrm{~d} v
\end{aligned}
$$

Observe that the two expressions for $g_{1}(u)$ in Equation (16) are not only generalized Krätzel integrals but they are also statistical densities of a product. We can evaluate the explicit form of the 
density by using arbitrary moments and then inverting the expression. Consider the $(s-1)$ th moments of $x_{1}$ and $x_{2}$. Then, $E\left[x_{1} x_{2}\right]^{s-1}=E\left[x_{1}^{s-1}\right] E\left[x_{2}^{s-1}\right]$ due to statistical independence, where $E[\cdot]$ denotes the expected value of $[\cdot]$. That is,

$$
E\left[x_{j}^{s-1}\right]=\int_{0}^{\infty} x_{j}^{s-1} f_{j}\left(x_{j}\right) \mathrm{d} x_{j}=M_{f_{j}}(s), j=1,2
$$

whenever the expected values exist, where $M_{f_{j}}(s)$ is the Mellin transform of the density $f_{j}$, with Mellin parameter $s$, when this Mellin transform exists. Evaluating $E\left[x_{j}^{s-1}\right]$ for the density in Equation (15), we have the following:

$$
E\left[x_{j}^{s-1}\right]=\frac{a_{j}^{-\frac{(s-1)}{\delta_{j}}} \Gamma\left(\frac{\gamma_{j}+s-1}{\delta_{j}}\right)}{\Gamma\left(\frac{\gamma_{j}}{\delta_{j}}\right)}, \Re\left(\gamma_{j}+s-1\right)>0, j=1,2 .
$$

Observe that in Equation (17) the explicit form of the normalizing constant $c_{j}$ is used, $c_{j}$ is such that $E\left[x_{j}^{s-1}\right]=1$ when $s=1$. Then, taking the product

$$
E\left[u^{s-1}\right]=\left\{\prod_{j=1}^{2} \frac{a_{j}^{\frac{1}{\delta_{j}}}}{\Gamma\left(\frac{\gamma_{j}}{\delta_{j}}\right)}\right\}\left\{\prod_{j=1}^{2} \Gamma\left(\frac{\gamma_{j}-1}{\delta_{j}}+\frac{s}{\delta_{j}}\right) a_{j}^{-\frac{s}{\delta_{j}}}\right\},
$$

for $\Re\left(\gamma_{j}+s-1\right)>0, j=1,2$. Then, the density $g_{1}(u)$ is available from the inverse Mellin transform or by inverting Equation (18). That is,

$$
\begin{aligned}
g_{1}(u) & =C \frac{1}{2 \pi i} \int_{c-i \infty}^{c+i \infty}\left\{\prod_{j=1}^{2} \Gamma\left(\frac{\gamma_{j}-1}{\delta_{j}}+\frac{s}{\delta_{j}}\right)\right\}\left(a_{1}^{\frac{1}{\delta_{1}}} a_{2}^{\frac{1}{\delta_{2}}} u\right)^{-s} \mathrm{~d} s \\
& =C H_{0,2}^{2,0}\left[\left.a_{1}^{\frac{1}{\delta_{1}}} a_{2}^{\frac{1}{\delta_{2}}} u\right|_{\left.\left(\frac{\gamma_{1}-1}{\delta_{1}}, \frac{1}{\delta_{1}}\right),\left(\frac{\gamma_{2}-1}{\delta_{2}}, \frac{1}{\delta_{2}}\right)\right],}\right. \\
C & =\prod_{j=1}^{2} \frac{a_{j}^{\frac{1}{\delta_{j}}}}{\Gamma\left(\frac{\gamma_{j}}{\delta_{j}}\right)} .
\end{aligned}
$$

Note that Equation (19) is the explicit form of the Krätzel integral as well as the statistical density $g_{1}(u)$. Instead of generalized gamma density for $f_{j}\left(x_{j}\right)$, suppose that the density of $x_{1}$ is a type- 1 beta density with the parameters $(\gamma+1, \alpha)$ and $f_{2}\left(x_{2}\right)$ is an arbitrary density then $f_{1}$ is of the form

$$
f_{1}\left(x_{1}\right)=\frac{\Gamma(\alpha+\gamma+1)}{\Gamma(\gamma+1) \Gamma(\alpha)} x_{1}^{\gamma}\left(1-x_{1}\right)^{\alpha-1}, 0 \leq x_{1} \leq 1, \alpha>0, \gamma>-1 .
$$

Usually, the parameters in a statistical density are real. Then, $g_{1}(u)$ becomes the following:

$$
\begin{aligned}
g_{1}(u) & =\int_{v} \frac{1}{v} f_{1}\left(\frac{u}{v}\right) f_{2}(v) \mathrm{d} v \\
& =\frac{\Gamma(\alpha+\gamma+1)}{\Gamma(\gamma+1) \Gamma(\alpha)} \int_{v \geq u} \frac{1}{v}\left(\frac{u}{v}\right)^{\gamma}\left(1-\frac{u}{v}\right)^{\alpha-1} f(v) \mathrm{d} v \\
& =\frac{\Gamma(\gamma+\alpha+1)}{\Gamma(\gamma+1)} \frac{u^{\gamma}}{\Gamma(\alpha)} \int_{v \geq u} v^{-\gamma-\alpha}(v-u)^{\alpha-1} f(v) \mathrm{d} v \\
& =\frac{\Gamma(\alpha+\gamma+1)}{\Gamma(\gamma+1)} K_{2, \gamma}^{-\alpha} f, \alpha>0, \gamma>-1
\end{aligned}
$$


where $K_{2, \gamma}^{-\alpha} f$ is Erdélyi-Kober fractional integral of the second kind of order $\alpha$ and parameter $\gamma$. From Equation (20), note that this fractional integral is a constant multiple of a statistical density of a product of positive random variables also. For generalizations of this result for the matrix-variate case, in real and complex domains, see [8]. By taking the density of a ratio of real scalar positive random variables, where the variables are independently distributed, with $x_{1}$ having a type-1 beta density with the parameters $(\gamma, \alpha)$ and $x_{2}$ having an arbitrary density we can show that the density of the ratio $u=\frac{x_{2}}{x_{1}}$ will produce a constant multiple of Erdélyi-Kober fractional integral of the first kind of order $\alpha$ and parameter $\gamma$, details or the generalizations of this result may be seen [8].

\section{Krätzel Integral and Bayesian Structures}

In a simple Bayesian structure in Bayesian statistical analysis, we have a conditional density of a random variables $x$, conditioned on a parameter $\theta$, or written as $f_{1}(x \mid \theta)$ or the density of $x$, given $\theta$. Then, $\theta$ has its own marginal density denoted by $f_{2}(\theta)$. Then, the joint density of $x$ and $\theta$ is $f_{1}(x \mid \theta) f_{2}(\theta)$. When both $x$ and $\theta$ are continuous variables, we call this situation as a continuous mixture. When one variable is discrete and the other continuous, we call it simply a mixture density. Then, the unconditional density of $x$, denoted by $f(x)$, is given by

$$
f(x)=\int_{\theta} f_{1}(x \mid \theta) f_{2}(\theta) \mathrm{d} \theta .
$$

A general format of the structure in Equation (21) is of the following type:

$$
f\left(x_{1}\right)=\int_{x_{2}} \ldots \int_{x_{k}} f_{1}\left(x_{1} \mid x_{2}, \ldots, x_{k}\right) f_{2}\left(x_{2} \mid x_{3}, \ldots, x_{k}\right) \ldots f_{k-1}\left(x_{k-1} \mid x_{k}\right) f_{k}\left(x_{k}\right) \mathrm{d} x_{2} \wedge \ldots \wedge \mathrm{d} x_{k} .
$$

For an application of this type of unconditional density for $k=3$, see [9]. When all the densities involved in Equations (21) and (22) are continuous, we also call Equations (21) and (22) as continuous mixtures. Consider Equation (21), where

$$
f_{1}(x \mid \theta)=\frac{\theta^{\gamma \delta}}{\Gamma(\gamma)} x^{\gamma-1} \mathrm{e}^{-\theta^{\delta} x}, x \geq 0, \theta>0, \delta>0, \gamma>0
$$

and

$$
f_{2}(\theta)=\frac{\rho b^{\frac{\alpha}{\rho}}}{\Gamma\left(\frac{\alpha}{\rho}\right)} \theta^{-\alpha-1} \mathrm{e}^{-b \theta^{-\rho}}, b>0, \alpha>0, \rho>0, \theta>0
$$

so that

$$
f_{1}(x \mid \theta) f_{2}(\theta)=\frac{\rho b^{\frac{\alpha}{\rho}}}{\Gamma(\gamma) \Gamma\left(\frac{\alpha}{\rho}\right)} x^{\gamma-1} \theta^{\gamma \delta-\alpha-1} \mathrm{e}^{-x \theta^{\delta}-b \theta^{-\rho}} .
$$

Then, the unconditional density is the following, denoting $\theta=v$ in the integral and denoting the unconditional density of $x$, again by $f(x)$ :

$$
f(x)=C_{1} \int_{v=0}^{\infty} v^{\gamma \delta-\alpha-1} \mathrm{e}^{-x v^{\delta}-b v^{-\rho}} \mathrm{d} v
$$

where

$$
C_{1}=\frac{\rho b^{\frac{\alpha}{\rho}}}{\Gamma(\gamma) \Gamma\left(\frac{\alpha}{\rho}\right)} x^{\gamma-1}, \alpha>0, \rho>0, \delta>0, \gamma>0, \rho>0, x>0 .
$$

Observe that Equation(23) is of the same structure of the Krätzel integral of Equation (2) of Section 1. Note that, if we use the general structure in Equation (22) and consider all densities as generalized gamma densities, then we obtain a generalization and extension of Krätzel integral to a multivariate situation. Such generalizations is considered below in this paper. 


\section{Pathway Extension of Krätzel Integral}

The author of [10] introduced a pathway model for rectangular matrix-variate case. By using a pathway parameter there, one can go to three different families of functions. When a model is fitted to a given data, then one member from the pathway family is sure to fit the data if the data fall into one of the three wide families of functions or in the transitional stages of going from one family to another family. The pathway model for real positive scalar variable situation is the following:

$$
f_{3}(x)=c_{3} x^{\gamma-1}\left[1+a(\alpha-1) x^{\delta}\right]^{-\frac{\eta}{\alpha-1}}, x>0, \alpha>1, \eta>0, \delta>0, a>0 .
$$

When $\alpha<1$, then we can write $\alpha-1=-(1-\alpha)$ so that the model in (24) switches to the model

$$
f_{4}(x)=c_{4} x^{\gamma-1}\left[1-a(1-\alpha) x^{\delta}\right]^{\frac{\eta}{1-\alpha}}, \alpha<1, \eta>0, a>0, \delta>0
$$

and, further, $1-a(1-\alpha) x^{\delta}>0$ in order to create statistical density out of $f_{4}(x)$. Its support is finite or it is a finite-range density, whereas in Equation (24) it is of infinite range and $x>0$ there. When $\alpha \rightarrow 1$, both Equations (24) and (25) go to the model

$$
f_{5}(x)=c_{5} x^{\gamma-1} \mathrm{e}^{-a \eta x^{\delta}}, a>0, x>0, \delta>0, \eta>0 .
$$

Thus, through the pathway parameter $\alpha$ one can move among the three families of functions $f_{j}(x), j=3,4,5$. Both Equations (24) and (25) can be taken as extensions of Equation (26). If Equation (26) is the ideal or stable situation in a physical system, then the unstable neighborhoods are given by Equations (24) and (25). The movement of $\alpha$ also describes the transitional stages. For the properties, generalizations and extension of the pathway model, see [11].The model in Equation (25) for $\gamma=1, a=1, \eta=1$ and for $\alpha<1, \alpha>1, \alpha \rightarrow 1$ is Tsallis' statistics in non-extensive statistical mechanics [12]. Some properties and other aspects of the pathway model see [11,13]. The model in Equation (24) for $a=1, \eta=1, \alpha>1, \alpha \rightarrow 1$ is superstatistics (see [14]). Superstatistics considerations come from the unconditional density described in Section 4 when the conditional and marginal densities belong to the exponential and gamma families of densities. Consider the model in Equation (24) with different parameters, take $f_{1}$ and $f_{2}$ of Section 1, and consider Mellin convolutions. Let $f_{31}$ and $f_{32}$ be two densities belonging to Equation (24) with different parameters. That is, let

$$
f_{3 j}\left(x_{j}\right)=c_{3 j} x_{j}^{\gamma_{j}-1}\left[1+a_{j}\left(\alpha_{j}-1\right) x_{j}^{\delta_{j}}\right]^{-\frac{\eta_{j}}{\alpha_{j}-1}}, x_{j}>0, \alpha_{j}>1, a_{j}>0, \gamma_{j}>0, \delta_{j}>0
$$

for $j=1,2$. Let $u=x_{1} x_{2}, v=x_{1}$. Consider the Mellin convolution of a product or let $x_{j}>0, j=1,2$ be independently distributed real scalar positive random variables with the densities $f_{31}$ and $f_{32}$ of (27) respectively. Then, the density of $u=x_{1} x_{2}$, denoted by $g_{p}(u)$, where $p$ stands for the pathway model, is the following:

$$
\begin{aligned}
g_{p}(u) & =\int_{v} \frac{1}{v} f_{31}(v) f_{32}\left(\frac{u}{v}\right) \mathrm{d} v \\
(G) & =c_{31} c_{32} u^{\gamma_{2}-1} \int_{v=0}^{\infty} v^{\gamma_{1}-\gamma_{2}-1}\left[1+a_{1}\left(\alpha_{1}-1\right) v^{\delta_{1}}\right]^{-\frac{\eta_{1}}{\alpha_{1}-1}} \\
& \times\left[1+a_{2}\left(\alpha_{2}-1\right)\left(\frac{u}{v}\right)^{\delta_{2}}\right]^{-\frac{\eta_{2}}{\alpha_{2}-1}} \mathrm{~d} v
\end{aligned}
$$

for $\alpha_{j}>1, a_{j}>0, \delta_{j}>0, \eta_{j}>0, j=1,2$. See also the versatile integral discussed in [15]. Various types of extensions of Krätzel integrals are involved in Equation (28). When $\alpha_{1} \rightarrow 1$, the first factor or the density in $(G)$ goes to the exponential form whereas the second part in Equation (28) remains in the type- 2 beta family form. This is one extension. In addition, when $\alpha_{2} \rightarrow 1$, the second part density in Equation (28) goes to the exponential form whereas the first part remains in the type-2 beta family 
of functions. When $\alpha_{1} \rightarrow 1$ and $\alpha_{2} \rightarrow 1$, Equation (28) goes to the format of the Krätzel integral in Equation (2) of Section 1. A model of the form in Equation (28) for the cases $\alpha_{j}<1, \alpha_{j}>1, \alpha_{j} \rightarrow 1$, individually, is studied in detail in [15].

Connection to Kobayashi Integrals

In Equation (28), let $\alpha_{1} \rightarrow 1$ and $\alpha_{2}$ remain the same. Then, Equation (28) reduces to the following form:

$$
\begin{aligned}
g_{p}(u) & =c_{31} c_{32} u^{\gamma_{2}-1} \int_{v=0}^{\infty} v^{\gamma_{1}-\gamma_{2}-1} \mathrm{e}^{-a_{1} \eta_{1} v^{\delta_{1}}} \\
& \times\left[1+a_{2}\left(\alpha_{2}-1\right)\left(\frac{u}{v}\right)^{\delta_{2}}\right]^{-\frac{\eta_{2}}{\alpha_{2}-1}} \mathrm{~d} v .
\end{aligned}
$$

Observe that Equation (29) is a more general form of ultra gamma integral and Kobayashi integral. The Kobayashi form is available from the Mellin convolution of a ratio. Let $u_{1}=\frac{x_{2}}{x_{1}}$ with $x_{1}=v$, and let $x_{1}$ and $x_{2}$ be independently distributed pathway random variables as described in Section 5 . Then, $x_{1}=v, x_{2}=u_{1} v$ and $\mathrm{d} x_{1} \wedge \mathrm{d} x_{2}=v \mathrm{~d} u_{1} \wedge \mathrm{d} v$. Then, the pathway density of $u_{1}$, denoted by $g_{p 1}\left(u_{1}\right)$, is the following for $\alpha_{1} \rightarrow 1$ :

$$
\begin{aligned}
g_{p 1}\left(u_{1}\right) & =c_{31} c_{32} u^{\gamma_{2}-1} \int_{v=0}^{\infty} v^{\gamma_{1}+\gamma_{2}-1} \mathrm{e}^{-a_{1} \eta_{1} v^{\delta_{1}}} \\
& \times\left[1+a_{2}\left(\alpha_{2}-1\right)\left(u_{1} v\right)^{\delta_{2}}\right]^{-\frac{\eta_{2}}{\alpha_{2}-1}}
\end{aligned}
$$

for $a_{j}>0, \gamma_{j}>0, \delta_{j}>0, \eta_{j}>0, j=1,2, \alpha_{2}>1$. Kobayashi integral is obtained from Equation (30) by putting $a_{2}\left(\alpha_{2}-1\right) u_{1}^{\delta_{2}}=\lambda$ and $\frac{\eta_{2}}{\alpha_{2}-1}=\eta$, (see [16,17]). Some people call Kobayashi form as ultra gamma integral. Observe that Equation (30) is a much more general and flexible format and for varying $\alpha_{2}$ we have three families of functions in Equation (30) including Kobayashi format. The Mellin transform of $g_{p 1}\left(u_{1}\right)$, with Mellin parameter $s$, is available from $u_{1}=\frac{x_{2}}{x_{1}}$ form, namely

$$
M_{g_{p 1}}(s)=M_{f_{1}}(2-s) M_{f_{2}}(s) \text { or } E\left[u_{1}^{s-1}\right]=E\left[x_{1}^{-s+1}\right] E\left[x_{2}^{s-1}\right]
$$

and these moments are available from the pathway densities of $x_{1}$ and $x_{2}$ with $\alpha_{1} \rightarrow 1$.

\section{Multivariate Extensions of Krätzel Integrals}

Let us start with the case of three variables. Let $x_{j}>0, j=1,2,3$ be three real scalar variables and let the associated functions be $f_{j}\left(x_{j}\right), j=1,2,3$, respectively. If $x_{j}>0, j=1,2,3$ are real scalar random variables, independently distributed, then $f_{j}\left(x_{j}\right), j=1,2,3$ may be the corresponding densities. Let $u=x_{1} x_{2} x_{3}$ be the product and let $v=x_{2} x_{3}, w=x_{3}$. Then, $x_{1} \wedge \mathrm{d} x_{2} \wedge \mathrm{d} x_{3}=\frac{1}{v w} \mathrm{~d} u \wedge \mathrm{d} v \wedge \mathrm{d} w$. Mellin convolution of a product involving three real scalar variables is considered in [18]. Let

$$
f_{j}\left(x_{j}\right)=c_{j} x_{j}^{\gamma_{j}-1} \mathrm{e}^{-a_{j} x_{j}^{\delta_{j}}}, a_{j}>0, \delta_{j}>0, \gamma_{j}>0, j=1,2,3
$$

where $c_{j}$ is a constant and it may be normalizing constant if $f_{j}$ in Equation (31) is a density. Then, the density of $u$ or Mellin convolution of the product, again denoted by $g(u)$, is the following:

$$
\begin{aligned}
g(u) & =\int_{v} \int_{w} \frac{1}{v w} f_{1}\left(\frac{u}{v w}\right) f_{2}\left(\frac{v}{w}\right) f_{3}(w) \mathrm{d} v \wedge \mathrm{d} w \\
& =c_{1} c_{2} c_{3} \int_{v} \int_{w} \frac{1}{v w}\left(\frac{u}{v}\right)^{\gamma_{1}-1}\left(\frac{v}{w}\right)^{\gamma_{2}-1} w^{\gamma_{3}-1} \\
& \times \mathrm{e}^{-a_{1}\left(\frac{u}{v}\right)^{\delta_{1}}-a_{2}\left(\frac{v}{w}\right)^{\delta_{2}}-a_{3} w^{\delta_{3}}} \mathrm{~d} v \wedge \mathrm{d} w
\end{aligned}
$$


where Equation (32) is the general structure whatever be the $f_{j}$ s, and Equation (33) is the case when $f_{j}$ s belong to Equation (31). Then, Equation (33) can be taken as a bivariate version of the Krätzel integral. Observe that in the exponent we have $v$ and $w$ with positive and negative exponents. If we take $u=x_{1} x_{2} x_{3}, v=x_{2}, w=x_{3}$, then the exponential part in $g(u)$ is of the following form:

$$
\mathrm{e}^{-a_{1}\left(\frac{u}{\partial w}\right)^{\delta_{1}}-a_{2} v^{\delta_{2}}-a_{3} w^{\delta_{3}}} .
$$

In the format of Equation (33), we can take $v=x_{1} x_{2}, w=x_{2}$ or $v=x_{2} x_{3}, w=x_{1}$. These produce two more different forms corresponding to Equation (33). We can also take $u=x_{1} x_{2} x_{3}=u_{12} x_{3}, u_{12}=$ $x_{1} x_{2}$. We can get the density of $u_{12}$ first by using $f_{1}$ and $f_{2}$. Let the density of $u_{12}$ be denoted as $g_{12}\left(u_{12}\right)$. Then, by using $g_{12}$ and $f_{3}$, we can get the density of $u$. This produces another bivariate extension of the Krätzel integral. Follow the same procedure by taking $u=u_{23} x_{1}, u_{13} x_{2}$ where $u_{23}=x_{2} x_{3}, u_{13}=x_{1} x_{3}$. In these cases, obtain the densities of $u_{13}$ and $u_{23}$ first and then proceed. These produce other different bivariate extensions of Krätzel integrals. For example, let $u=x_{1} x_{2} x_{3}=u_{12} x_{3}, u_{12}=x_{1} x_{2}$. Let the density of $u_{12}$ be $g_{12}\left(u_{12}\right)$. Then, from the two-variables case,

$$
\text { (H) } g_{12}\left(u_{12}\right)=\int_{v} \frac{1}{v} f_{1}\left(\frac{u_{12}}{v}\right) f_{2}(v) \mathrm{d} v .
$$

Let the density of $u$ be $g(u)$. Then,

$$
\text { (I) } \begin{aligned}
g(u) & =\int_{w} \frac{1}{w} g_{12}\left(\frac{u}{u_{12}}\right) f_{3}(w) \mathrm{d} w \\
& =\int_{w} \frac{1}{w}\left[\int_{v} \frac{1}{v} f_{1}\left(\frac{u_{12}}{v}\right) f_{2}(v) \mathrm{d} v\right] f_{3}(w) \mathrm{d} w \\
& =\int_{v} \int_{w} \frac{1}{v w} f_{1}\left(\frac{u_{12}}{v}\right) f_{2}(v) f_{3}(w) \mathrm{d} v \wedge \mathrm{d} w .
\end{aligned}
$$

However, we also have

$$
\text { (J) } g_{12}\left(u_{12}\right)=\int_{v} \frac{1}{v} f_{1}(v) f_{2}\left(\frac{u_{12}}{v}\right) \mathrm{d} v .
$$

Substituting for $g_{12}$ from $(J)$ into $(H)$, we have the following and other forms from the symmetry also:

$$
\begin{aligned}
g(u) & =\int_{w} \frac{1}{w}\left[\int_{v} \frac{1}{v} f_{1}(v) f_{2}\left(\frac{u}{v}\right) \mathrm{d} v\right] f_{3}(w) \mathrm{d} w \\
(K) & =\int_{v} \int_{w} \frac{1}{v w} f_{1}(v) f_{2}\left(\frac{u}{v}\right) f_{3}(w) \mathrm{d} v \wedge \mathrm{d} w \\
& =\int_{v} \int_{w} \frac{1}{v w} f_{1}(v) f_{2}(w) f_{3}\left(\frac{u}{v}\right) \mathrm{d} v \wedge \mathrm{d} w \\
& =\int_{v} \int_{w} \frac{1}{v w} f_{1}\left(\frac{u}{v}\right) f_{2}(w) f_{3}(v) \mathrm{d} v \wedge \mathrm{d} w \\
& =\int_{v} \int_{w} \frac{1}{v w} f_{1}(w) f_{2}(v) f_{3}\left(\frac{u}{v}\right) \mathrm{d} v \wedge \mathrm{d} w \\
& =\int_{v} \int_{w} \frac{1}{v w} f_{1}(w) f_{2}\left(\frac{u}{v}\right) f_{3}(v) \mathrm{d} v \wedge \mathrm{d} w .
\end{aligned}
$$

A few such forms, as in $(K)$, are described in [7] and hence these are not repeated here. From the products of four or more variables $x_{j}>0, j=4,5, \ldots, k$, we can have several different extensions of Krätzel integral for bivariate, trivariate and general multivariate cases. The method is similar to what is explained above and hence further discussion is omitted. Even though hundreds of different integral 
representations are available for the density of $u=x_{1} \ldots x_{k}$, the explicit evaluation of the density $g(u)$ of $u$ is possible by inverting the corresponding Mellin transform, namely

$$
M_{g}(s)=\prod_{j=1}^{k} M_{f_{j}}(s)
$$

and take the inverse Mellin transform of $\prod_{j=1}^{k} M_{f_{j}}(s)$ to obtain the density $g$ of $u=x_{1} x_{2} \ldots x_{k}$.

\section{Connections to Fractional Integrals}

Let $x_{j}>0, j=1,2,3$ be real scalar random variables, independently distributed with densities $f_{j}\left(x_{j}\right), j=1,2,3$, respectively. Let $u=x_{1} x_{2} x_{3}, v=x_{2}, w=x_{3}$. Then, $\mathrm{d} x_{1} \wedge \mathrm{d} x_{2} \wedge \mathrm{d} x_{3}=\frac{1}{v w} \mathrm{~d} u \wedge \mathrm{d} v \wedge$ $\mathrm{d} w$. Let $f_{1}$ be a real scalar type- 1 beta density with the parameters $(\gamma+1, \alpha)$, or with the density:

$$
f_{1}\left(x_{1}\right)=\frac{\Gamma(\gamma+1+\alpha)}{\Gamma(\gamma+1) \Gamma(\alpha)} x_{1}^{\gamma}\left(1-x_{1}\right)^{\alpha-1}, 0 \leq x_{1} \leq 1, \alpha>0, \gamma>-1 .
$$

Let $f_{2}$ and $f_{3}$ be arbitrary densities. Then,

$$
f_{1}\left(x_{1}\right)=f\left(\frac{u}{v w}\right)=\frac{\Gamma(\gamma+1+\alpha)}{\Gamma(\gamma+1) \Gamma(\alpha)}\left(\frac{u}{v w}\right)^{\gamma}\left(1-\frac{u}{v w}\right)^{\alpha-1} .
$$

Then, the density of $u$ from (34), $f_{2}$ and $f_{3}$, denoted again by $g(u)$, is the following:

$$
\begin{aligned}
g(u) & =\frac{\Gamma(\gamma+1+\alpha)}{\Gamma(\gamma+1)} \frac{u^{\gamma}}{\Gamma(\alpha)} \int_{v} \int_{w}(v w)^{-\gamma-\alpha}(v w-u)^{\alpha-1} f_{2}(v) f_{3}(w) \mathrm{d} v \wedge \mathrm{d} w \\
& =\frac{\Gamma(\gamma+1+\alpha)}{\Gamma(\gamma+1)} K_{2, \gamma}^{-\alpha}\left(f_{2}, f_{3}\right) .
\end{aligned}
$$

If $f_{3}$ and the corresponding $w$ are absent, then $K_{2, \gamma}^{-\alpha}\left(f_{2}, f_{3}\right)=K_{2, \gamma}^{-\alpha} f_{2}$ which is Erdélyi-Kober fractional integral of the second kind and of order $\alpha$ and parameter $\gamma$ where the arbitrary function is $f_{2}$. Similarly, when $f_{2}$ and $v$ are absent, we get Erdélyi-Kober fractional integral of the second kind of order $\alpha$ and parameter $\gamma$ with the arbitrary function $f_{3}$. Hence, Equation (35) is a bivariate generalization of Erdélyi-Kober fractional integral of the second kind. This generalization in Equation (35) is different from the multivariate case of Mathai [8] and multi-index case of Kiryakova [19]. Other extension to bivariate case of fractional integrals are available from the various representations in $(K)$ of Section 6 by taking one or two, out of the three functions there, as real scalar type- 1 beta densities.

Let $u_{1}=\frac{x_{1}}{x_{2}}$ with $x_{1}=v$ so that $x_{2}=\frac{v}{u_{1}}$ and $\mathrm{d} x_{1} \wedge \mathrm{d} x_{2}=-\frac{v}{u_{1}^{2}} \mathrm{~d} u_{1} \wedge \mathrm{d} v$. Then, the density of $u_{1}$, denoted by $g_{1}\left(u_{1}\right)$, is the following:

$$
g_{1}\left(u_{1}\right)=\int_{v} \frac{v}{u_{1}^{2}} f_{1}(v) f_{2}\left(\frac{v}{u_{1}}\right) \mathrm{d} v
$$

Let $f_{1}(v)=f(v)$, be an arbitrary density and let $f_{2}\left(x_{2}\right)$ be a real scalar type-1 beta density with the parameters $(\gamma, \alpha)$. Then, from Equation (36),

$$
\begin{aligned}
g_{1}\left(u_{1}\right) & =\frac{\Gamma(\gamma+\alpha)}{\Gamma(\gamma) \Gamma(\alpha)} \int_{v} \frac{v}{u_{1}^{2}} f(v)\left(\frac{v}{u_{1}}\right)^{\gamma-1}\left(1-\frac{v}{u_{1}}\right)^{\alpha-1} \mathrm{~d} v \\
& =\frac{\Gamma(\gamma+\alpha)}{\Gamma(\gamma)} \frac{u_{1}^{-\alpha-\gamma}}{\Gamma(\alpha)} \int_{v \leq u_{1}} v^{\gamma}(u-v)^{\alpha-1} f(v) \mathrm{d} v \\
& =\frac{\Gamma(\gamma+\alpha)}{\Gamma(\gamma)} K_{1, \gamma}^{-\alpha} f
\end{aligned}
$$


where $K_{1, \gamma}^{-\alpha} f$ is Erdélyi-Kober fractional integral of the first kind of order $\alpha$ and parameter $\gamma$. Consider the generalization to three variables. Let $u_{1}=\frac{x_{2} x_{3}}{x_{1}}, x_{2}=v, x_{3}=w \Rightarrow x_{1}=\frac{v w}{u_{1}}$. Then, $\mathrm{d} x_{1} \wedge \mathrm{d} x_{2} \wedge$ $\mathrm{d} x_{3}=-\frac{v w}{u_{1}^{2}} \mathrm{~d} u_{1} \wedge \mathrm{d} v \wedge \mathrm{d} w$ and the marginal density of $u_{1}$, again denoted by $g_{1}\left(u_{1}\right)$, is the following:

$$
\begin{aligned}
g_{1}\left(u_{1}\right) & =\frac{\Gamma(\gamma+\alpha)}{\Gamma(\gamma) \Gamma(\alpha)} \int_{v} \int_{w} \frac{v w}{u_{1}^{2}}\left(\frac{v w}{u_{1}}\right)^{\gamma-1}\left(1-\frac{v w}{u_{1}}\right)^{\alpha-1} f_{2}(v) f_{3}(w) \mathrm{d} v \wedge \mathrm{d} w \\
& =\frac{\Gamma(\gamma+\alpha)}{\Gamma(\gamma)} \frac{u_{1}^{-\gamma-\alpha}}{\Gamma(\alpha)} \int_{v} \int_{w}(v w)^{\gamma}\left(u_{1}-v w\right)^{\alpha-1} f_{2}(v) f_{3}(w) \mathrm{d} v \wedge \mathrm{d} w \\
& =\frac{\Gamma(\gamma+\alpha)}{\Gamma(\gamma)} K_{1, \gamma}^{-\alpha}\left(f_{1}, f_{2}\right)
\end{aligned}
$$

where $K_{1, \gamma}^{-\alpha}\left(f_{2}, f_{3}\right)$ of Equation (38) may be called Erdélyi-Kober fractional integral of the first kind of order $\alpha$ and parameter $\gamma$ in the bivariate case or with two arbitrary functions. Here, the integrals are over $0 \leq v \leq 1,0 \leq w \leq 1,0 \leq v w \leq u_{1}$. This type of generalization is different from the ones available in the literature. Various definitions of fractional integrals, fractional derivatives, and fractional differentials equations and their properties may be seen in [20-22].

\section{Krätzel Integral in the Real Matrix-variate Case}

It is easier to interpret Krätzel integral in terms of statistical distributions. Let $X_{1}$ and $X_{2}$ be two $p \times p$ real positive definite matrix random variables with the densities $f_{1}\left(X_{1}\right)$ and $f_{2}\left(X_{2}\right)$, respectively. Density here means a real-valued scalar function $f(X)$ of the positive definite matrix $X>O$, such that $f(X) \geq 0$ for all $X>O$ and $\int_{X>O} f(X) \mathrm{d} X=1$. That is, for $X_{j}>0, j=1,2$ ( positive definite), $f_{j}\left(X_{j}\right) \geq 0$ for all $X_{j}>O$ and $\int_{X_{j}>O} f_{j}\left(X_{j}\right) \mathrm{d} X_{j}=1, j=1,2$. Let $X_{j}>O$ have a real matrix-variate gamma density. That is,

$$
f_{j}\left(X_{j}\right)=\frac{\left|A_{j}\right|^{\gamma_{j}}}{\Gamma_{p}\left(\gamma_{j}\right)}\left|X_{j}\right|^{\gamma_{j}-\frac{p+1}{2}} \mathrm{e}^{-\operatorname{tr}\left(A_{j} X_{j}\right)}, X_{j}>O, A_{j}>O, \Re\left(\gamma_{j}\right)>\frac{p-1}{2}, j=1,2
$$

where, in Equation (39), $A_{j}>\mathrm{O}$ is a $p \times p$ real positive definite constant matrix for $j=1,2 .$. When $p=1$, we have the corresponding scalar variable gamma density. The real matrix-variate gamma function $\Gamma_{p}\left(\gamma_{j}\right)$ is explained below. In the scalar case we have taken exponents $\delta_{j}>0, j=1,2$ but if we take exponents in the matrix-variate case then the transformations will not produce nice forms for further derivations, see the types of difficulties from [23], and hence we have taken $\delta_{1}=\delta_{2}=1$ in the matrix-variate case. Let us consider symmetric product $U=X_{2}^{\frac{1}{2}} X_{1} X_{2}^{\frac{1}{2}}$ where $X_{2}^{\frac{1}{2}}>O$ is the positive definite square root of the positive definite matrix $X_{2}>0$. We have taken the symmetric product because the transformations are on symmetric cases. Let $V=X_{2}$. Then, from Mathai [23], we can derive $\mathrm{d} X_{1} \wedge \mathrm{d} X_{2}=|V|^{-\frac{p+1}{2}} \mathrm{~d} U \wedge \mathrm{d} V$ and then proceeding as in the scalar variable case, the density of $U$, denoted again by $g(U)$, is given by the following:

$$
g(U)=\int_{V}|V|^{-\frac{p+1}{2}} f_{1}\left(V^{-\frac{1}{2}} U V^{-\frac{1}{2}}\right) f_{2}(V) \mathrm{d} V
$$

where $f_{1}$ and $f_{2}$ in Equation (40) are some general densities. Consider the case when $f_{j}\left(X_{j}\right)$ is a real matrix-variate gamma density given by the following:

$$
f_{j}\left(X_{j}\right)=\frac{\left|A_{j}\right|^{\gamma_{j}}}{\Gamma_{p}\left(\gamma_{j}\right)}\left|X_{j}\right|^{\gamma_{j}-\frac{p+1}{2}} \mathrm{e}^{-\operatorname{tr}\left(A_{j} X_{j}\right)}
$$


for $A_{j}>O, X_{j}>O, \Re\left(\gamma_{j}\right)>\frac{p-1}{2}, j=1,2$, where $\Gamma_{p}\left(\gamma_{j}\right)$ is the real matrix-variate gamma given by

$$
\Gamma_{p}(\alpha)=\pi^{\frac{p(p-1)}{4}} \Gamma(\alpha) \Gamma\left(\alpha-\frac{1}{2}\right) \ldots \Gamma\left(\alpha-\frac{p-1}{2}\right), \Re(\alpha)>\frac{p-1}{2} .
$$

For the densities in Equation (41), with $\Gamma_{p}\left(\gamma_{j}\right)$ defined in Equation (42), the density of $U$ is given by the following:

$$
g(U)=C|U|^{\gamma_{1}-\frac{p+1}{2}} \int_{V>O}|V|^{\gamma_{2}-\gamma_{1}-\frac{p+1}{2}} \mathrm{e}^{-\operatorname{tr}\left(V^{-\frac{1}{2}} A_{1} V^{-\frac{1}{2}} U\right)-\operatorname{tr}\left(A_{2} V\right)} \mathrm{d} V
$$

for $A_{j}>O, V>O, U>O, \Re\left(\gamma_{j}\right)>\frac{p-1}{2}, j=1,2$ where

$$
C=\prod_{j=1}^{2} \frac{\left|A_{j}\right|^{\gamma_{j}}}{\Gamma_{p}\left(\gamma_{j}\right)}
$$

This Equation (43) is the Krätzel integral in the real matrix-variate case. Note that, if $A_{1}$ is a positive scalar quantity, then it can be taken out of $V$ and then $V^{-1}$ will be obtained corresponding to the real scalar case.

The model in Equation (41) is also connected to Maxwell-Boltzmann and Raleigh densities in physics. Their matrix-variate, multivariate and rectangular matrix-variate extensions and some applications in reliability analysis are given in [24]. Their complex matrix-variate analogs can be worked out but they do not seem to be in print in the literature yet.

\section{Krätzel Integral in the Complex Matrix-variate Case}

Here, we consider $p \times p$ Hermitian positive definite matrices $\tilde{X}_{j}>0, j=1,2$ and Hermitian positive definite square root $\tilde{X}_{2}^{\frac{1}{2}}$. Consider the symmetric product $\tilde{U}=\tilde{X}_{2}^{\frac{1}{2}} \tilde{X}_{1} \tilde{X}_{2}^{\frac{1}{2}}, \tilde{V}=\tilde{X}_{2}$. Then, from [23] we have $\mathrm{d} \tilde{X}_{1} \wedge \mathrm{d} \tilde{X}_{2}=|\operatorname{det}(V)|^{-p} \mathrm{~d} \tilde{U} \wedge \mathrm{d} \tilde{V}$. Let the density of $\tilde{U}$ be denoted by $\tilde{g}(\tilde{U})$ when $\tilde{X}_{j}, j=1,2$ are independently distributed with the complex matrix-variate gamma densities given by

$$
\tilde{f}_{j}\left(\tilde{X}_{j}\right)=\frac{\left|\operatorname{det}\left(A_{j}\right)\right|^{\gamma_{j}}}{\tilde{\Gamma}_{p}\left(\gamma_{j}\right)}\left|\operatorname{det}\left(\tilde{X}_{j}\right)\right|^{\gamma_{j}-p} \mathrm{e}^{-\operatorname{tr}\left(A_{j} \tilde{X}_{j}\right)}, \tilde{X}_{j}>O, \Re\left(\gamma_{j}\right)>p-1, j=1,2
$$

where $\tilde{\Gamma}_{p}(\alpha)$ is the complex matrix-variate gamma given by the following:

$$
\tilde{\Gamma}_{p}(\alpha)=\pi^{\frac{p(p-1)}{2}} \Gamma(\alpha) \Gamma(\alpha-1) \ldots \Gamma(\alpha-p+1), \Re(\alpha)>p-1 .
$$

Then, from Equations (44) and (45), proceeding as in the real matrix-variate case the density of $\tilde{U}$, denoted by $\tilde{g}(\tilde{U})$, is the following:

$$
\tilde{g}(\tilde{U})=\tilde{C}|\operatorname{det}(\tilde{U})|^{\gamma_{1}-p} \int_{\tilde{V}>0}|\operatorname{det}(\tilde{V})|^{\gamma_{2}-\gamma_{1}-p} \mathrm{e}^{-\operatorname{tr}\left(\tilde{V}^{-\frac{1}{2}} A_{1} \tilde{V}^{-\frac{1}{2}} \tilde{U}\right)-\operatorname{tr}\left(A_{2} \tilde{V}\right)} \mathrm{d} \tilde{V}
$$

for $\Re\left(\gamma_{j}\right)>p-1, A_{j}>O, \tilde{V}>O, \tilde{U}>O, j=1,2$ where

$$
\tilde{C}=\prod_{j=1}^{2} \frac{\left|\operatorname{det}\left(A_{j}\right)\right|^{\gamma_{j}}}{\tilde{\Gamma}_{p}\left(\gamma_{j}\right)} .
$$

\section{Extension to Rectangular Matrix-variate Case}

Let $X=\left(x_{i j}\right)$ be a $p \times q, q \geq p$ matrix of full rank $p$ where the elements $x_{i j}$ s are distinct real scalar variables. Let $A>O$ be $p \times p$ and $B>O$ be $q \times q$ constant real positive definite matrices. Let a prime 
denote the transpose, let $\operatorname{tr}(\cdot)$ be the trace of $(\cdot)$, and let, for example, $A^{\frac{1}{2}}$ be the positive definite square root of the positive definite matrix $A>O$. Consider the model

$$
\begin{aligned}
f(X) & =C\left|A^{\frac{1}{2}} X B X^{\prime} A^{\frac{1}{2}}\right|^{\gamma}\left|I+a_{1}\left(q_{1}-1\right)\left(A^{\frac{1}{2}} X B X^{\prime} A^{\frac{1}{2}}\right)\right|^{-\frac{1}{q_{1}-1}} \\
& \times\left|I+a_{2}\left(q_{2}-1\right)\left(A^{\frac{1}{2}} X B X^{\prime} A^{\frac{1}{2}}\right)^{-1}\right|^{-\frac{1}{q_{2}-1}}
\end{aligned}
$$

for $a_{j}>0, q_{j}>1, j=1,2, \gamma>-\frac{q}{2}+\frac{p-1}{2}$. Observe that

$$
\lim _{q_{j} \rightarrow 1}\left|I+a_{j}\left(q_{j}-1\right)\left(A^{\frac{1}{2}} X B X^{\prime} A^{\frac{1}{2}}\right)\right|^{-\frac{1}{q_{j}-1}}=\mathrm{e}^{-\operatorname{tr}\left(A^{\frac{1}{2}} X B X^{\prime} A^{\frac{1}{2}}\right)}
$$

for $j=1$, 2. Let

$$
f_{1}(X)=\lim _{q_{1} \rightarrow 1} f(X), f_{2}(X)=\lim _{q_{2} \rightarrow 1} f(X), f_{3}(X)=\lim _{q_{1} \rightarrow 1, q_{2} \rightarrow 1} f(X) .
$$

Then,

$$
\begin{gathered}
f_{1}(X)=C_{1}\left|A^{\frac{1}{2}} X B X^{\prime} A^{\frac{1}{2}}\right|^{\gamma} \mathrm{e}^{-a_{1}\left(A^{\frac{1}{2}} X B X^{\prime} A^{\frac{1}{2}}\right)} \\
\times\left|I+a_{2}\left(q_{2}-1\right)\left(A^{\frac{1}{2}} X B X^{\prime} A^{\frac{1}{2}}\right)^{-1}\right|^{-\frac{1}{q_{2}-1}} \cdot \\
f_{2}(X)=C_{2}\left|A^{\frac{1}{2}} X B X^{\prime} A^{\frac{1}{2}}\right|^{\gamma}\left|I+a_{1}\left(q_{1}-1\right)\left(A^{\frac{1}{2}} X B X^{\prime} A^{\frac{1}{2}}\right)\right|^{-\frac{1}{q_{1}-1}} \\
\times \mathrm{e}^{-a_{2} \operatorname{tr}\left(A^{\frac{1}{2}} X B X^{\prime} A^{\frac{1}{2}}\right)^{-1}} . \\
f_{3}(X)=C_{3}\left|A^{\frac{1}{2}} X B X^{\prime} A^{\frac{1}{2}}\right|^{\gamma} \mathrm{e}^{-a_{1}\left(A^{\frac{1}{2}} X B X^{\prime} A^{\frac{1}{2}}\right)-a_{2}\left(A^{\frac{1}{2}} X B X^{\prime} A^{\frac{1}{2}}\right)^{-1}} .
\end{gathered}
$$

Then, $f_{3}(X)$, coming from Equations (46) and (47), is the real rectangular matrix-variate version of Krätzel integral. In a physical model building situation, if Equation (50) is the stable or ideal situation, then Equations (46), (48) and (49) describe the unstable neighborhoods. From the discussion in Sections 2 and 3, we can see that the model in Equations (46) and (48)-(50) can also be generated by M-convolution of product or density of a product in the real matrix-variate case. In Equation (50), for simplicity, we have taken the coefficient parameters as scalar quantities. We can evaluate the normalizing constants $C, C_{1}, C_{2}, C_{3}$ by using the following steps: Let

$$
\text { (L) } Y=A^{\frac{1}{2}} X B^{\frac{1}{2}} \Rightarrow \mathrm{d} X=|A|^{-\frac{p}{2}}|B|^{-\frac{q}{2}} \mathrm{~d} Y
$$

from the general linear transformation (see [23] for the Jacobian in $(L)$ and other Jacobians to follow). Let the corresponding function $f(X)$ be denoted by $f_{01}(Y)$. Then,

$$
\begin{aligned}
f_{01}(Y) & =C|A|^{-\frac{p}{2}}|B|^{-\frac{q}{2}}\left|Y Y^{\prime}\right|^{\gamma}\left|I+a_{1}\left(q_{1}-1\right)\left(Y Y^{\prime}\right)\right|^{-\frac{1}{q_{1}-1}} \\
& \times\left|I+a_{2}\left(q_{2}-1\right)\left(Y Y^{\prime}\right)^{-1}\right|^{-\frac{1}{q_{2}-1}} .
\end{aligned}
$$

Let the corresponding functions $f_{1}(X), f_{2}(X), f_{3}(X)$ be denoted by $f_{11}(Y), f_{21}(Y), f_{31}(Y)$, respectively. Note that $Y$ has $p q$ real scalar variables whereas $S=Y Y^{\prime}$, which is a $p \times p$ real positive 
definite matrix, has only $p(p+1) / 2$ elements. However, we can obtain a relationship between $\mathrm{d} Y$ and $\mathrm{d} S$ (see [23]). It is the following:

$$
(M) \mathrm{d} Y=\frac{\pi^{\frac{p q}{2}}}{\Gamma_{p}\left(\frac{q}{2}\right)}|S|^{\frac{q}{2}-\frac{p+1}{2}} \mathrm{~d} S
$$

where $Y$ in $(M)$ is $p \times q$, whereas $S$ is $p \times p$. Let the corresponding functions of $S$ be denoted by $f_{02}(S), f_{12}(S), f_{22}(S), f_{32}(S)$, respectively. Then, for example, $f_{02}(S)$ is the following:

$$
\begin{aligned}
f_{02}(S) & =C|A|^{-\frac{p}{2}}|B|^{-\frac{q}{2}}|S|^{\gamma+\frac{q}{2}-\frac{p+1}{2}}\left|I+a_{1}\left(q_{1}-1\right)(S)\right|^{-\frac{1}{q_{1}-1}} \\
& \times\left|I+a_{2}\left(q_{2}-1\right)(S)^{-1}\right|^{-\frac{1}{q_{2}-1}} .
\end{aligned}
$$

\subsection{Multivariate Situation}

In Equation (46) and Equations (48)-(50), let $p=1$ and $q>1$; then, $Y$ is $1 \times q$ and of the form $Y=\left(y_{1}, \ldots, y_{q}\right)$. Then, $Y Y^{\prime}=y_{1}^{2}+\ldots+y_{q}^{2}$. Then, for $p=1$, the constant matrix $A$ is $1 \times 1$ and let it be $a_{3}>0$. Then, from Equation (51),

$$
\begin{aligned}
f_{01} & =C a_{3}^{-\frac{1}{2}}|B|^{-\frac{q}{2}}\left(y_{1}^{2}+\ldots+y_{q}^{2}\right)^{\gamma}\left[1+a_{1}\left(q_{1}-1\right)\left(y_{1}^{2}+\ldots+y_{q}^{2}\right)\right]^{-\frac{1}{q_{1}-1}} \\
& \times\left[1+a_{2}\left(q_{2}-1\right)\left(y_{1}^{2}+\ldots+y_{q}^{2}\right)^{-1}\right]^{-\frac{1}{q_{2}-1}} .
\end{aligned}
$$

Then, $f_{31}$ becomes the following:

$$
\begin{aligned}
f_{31}(Y) & =C_{3} a_{3}^{-\frac{1}{2}}|B|^{-\frac{q}{2}}\left[\left(y_{1}^{2}+\ldots+y_{q}^{2}\right)\right]^{\gamma} \\
& \times \mathrm{e}^{-a_{1}\left(y_{1}^{2}+\ldots+y_{q}^{2}\right)-a_{2}\left(y_{1}^{2}+\ldots+y_{q}^{2}\right)^{-1}}
\end{aligned}
$$

for $-\infty<y_{j}<\infty, j=1, \ldots, q$. We may call Equation (52) as the multivariate version of the basic Krätzel integral and $f_{01}$ for $p=1$ as the pathway extended form of $f_{31}$ in Equation (52).

Note that for a general $p>1$ we do not take exponents for $\left(A^{\frac{1}{2}} X B X^{\prime} A^{\frac{1}{2}}\right)$ because in the general case matrix transformations create problems while computing the Jacobians. The types of problem is described in [23]. However, for the scalar cases in $f_{02}, f_{12}, f_{22}, f_{32}$, we can take arbitrary exponents. Hence, we have the general Krätzel integrals in the multivariate case as the following:

$$
\begin{aligned}
f_{33}(Y) & =C_{3} a_{3}^{-\frac{1}{2}}|B|^{-\frac{q}{2}}\left[\left(y_{1}^{2}+\ldots+y_{q}^{2}\right)\right]^{\gamma} \\
& \times \mathrm{e}^{-a_{1}\left(y_{1}^{2}+\ldots+y_{q}^{2}\right)^{\delta}-a_{2}\left(y_{1}^{2}+\ldots+y_{q}^{2}\right)^{-\rho}}
\end{aligned}
$$

for $\delta>0, \rho>0$. Corresponding exponents can be included in $f_{03}, f_{13}, f_{23}$ as well. For evaluating the normalizing constant, we can do the following steps. Make use of the transformation and Jacobian in $(M)$ for $p=1$. Then, $S=s$ is a scalar variable. Then, for $p=1$, Equation (53) becomes the following:

$$
f_{34}(s)=a_{3}^{-\frac{1}{2}}|B|^{-\frac{q}{2}} \frac{\pi^{\frac{q}{2}}}{\Gamma\left(\frac{q}{2}\right)} s^{\gamma+\frac{q}{2}-1} \mathrm{e}^{-a_{1} s^{\delta}-a_{2} s^{-\rho}} .
$$

Since $s$ is a real scalar variable here, one can use the scalar version of Mellin convolution of a product or density of product of Sections 2 and 3, go to the Mellin transforms to evaluate the normalizing constant. The same procedure works for all the models $f_{04}, f_{14}, f_{24}$ also. 
9.2. Evaluation of the Normalizing Constant

Let

$$
\int_{s=0}^{\infty} s^{\gamma+\frac{q}{2}-1} \mathrm{e}^{-a s^{\delta}-b s^{-\rho}} \mathrm{d} s=g(b) \text { say. }
$$

Let $M_{g}(t)$ be the Mellin transform of $g(b)$ with Mellin parameter $t$. Then,

$$
M_{g}(t)=\int_{0}^{\infty} b^{t-1}\left\{\int_{s=0}^{\infty} s^{\gamma+\frac{q}{2}-1} \mathrm{e}^{-a s^{\delta}-b s^{-\rho}} \mathrm{d} s\right\} \mathrm{d} b
$$

Evaluating the $b$-integral we have the following:

$$
\int_{0}^{\infty} b^{t-1} \mathrm{e}^{-b s^{-\rho}} \mathrm{d} b=\Gamma(t) s^{\rho t}, \text { for } \Re(t)>0 .
$$

Now, evaluating the s-integral, we have the following:

$$
\int_{0}^{\infty} s^{\gamma+\frac{q}{2}+\rho t-1} \mathrm{e}^{-a s^{\delta}} \mathrm{d} s=\frac{\Gamma\left(\frac{\gamma+\rho t+q / 2}{\delta}\right)}{\delta a^{\frac{\gamma+\rho t+q / 2}{\delta}}}, \Re(\gamma+\rho t+q / 2)>0 .
$$

That is,

$$
M_{g}(t)=\frac{1}{\delta s \frac{\gamma+q / 2}{\delta}} \Gamma(t) \Gamma\left(\frac{\gamma+q / 2}{\delta}+\frac{\rho}{\delta} t\right) a^{-\frac{\rho}{\delta} t} .
$$

By taking the inverse Mellin transform, we have $g(b)$ as the following:

$$
\begin{aligned}
g(b) & =\frac{1}{\delta a^{\frac{\gamma+q / 2}{\delta}}} \frac{1}{2 \pi i} \int_{c-i \infty}^{c+i \infty} \Gamma(t) \Gamma\left(\frac{\gamma+q / 2}{\delta}+\frac{\rho}{\delta} t\right)\left(b a^{\frac{\rho}{\delta}}\right)^{-t} \mathrm{~d} t \\
& =\frac{1}{\delta a^{\frac{\gamma+q / 2}{\delta}}} H_{0,2}^{2,0}\left[\left.b a^{\frac{\rho}{\delta}}\right|_{(0,1),\left(\frac{\gamma+q / 2}{\delta}, \frac{\rho}{\delta}\right)}\right]
\end{aligned}
$$

where $H(\cdot)$ is the H-function, see [5]. Then, the normalizing constant is the following:

$$
C=a_{3}^{\frac{1}{2}}|B|^{\frac{q}{2}} \frac{\Gamma\left(\frac{q}{2}\right)}{\pi^{\frac{q}{2}}} \frac{\delta a^{\frac{\gamma+q / 2}{\delta}}}{H_{0,2}^{2,0}\left[\left.b a^{\frac{\rho}{\delta}}\right|_{(0,1),\left(\frac{\gamma+q / 2}{\delta}, \frac{\rho}{\delta}\right)}\right]} .
$$

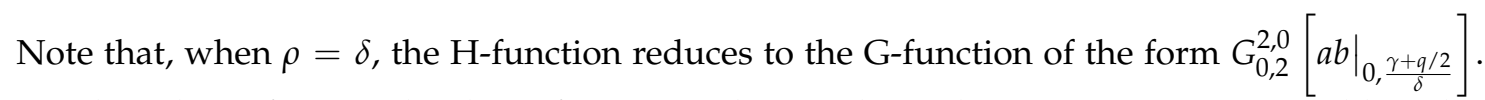
Then, replace the $\mathrm{H}$-function by the G-function. Observe that, when $p=1, A$ is $1 \times 1$ and let it be $a_{3}>0$. This is the $a_{3}$ appearing above.

Author Contributions: Conceptualization, A.M.M. and H.J.H.; methodology, A.M.M. and H.J.H.; validation, A.M.M. and H.J.H.; formal analysis, A.M.M. and H.J.H.; All authors have read and agreed to the published version of the manuscript.

Funding: This research received no external funding.

Acknowledgments: The authors would like to thank Francesco Mainardi for inviting us to contribute to this Special Issue. The authors would like to thank the reviewers for making valuable comments, which helped to improve the presentation in this paper.

Conflicts of Interest: The authors declare no conflict of interest in this paper.

\section{References}

1. Mathai, A.M.; Haubold, H.J. Erdélyi-Kober Fractional Calculus: From a Statistical Perspective, Inspired by Solar Neutrino Physics; Springer Briefs in Mathematical Physics: Singapore, 2018. 
2. Critchfield, C.L. Analytic forms of the thermonuclear function. In Cosmology, Fusion \& Other Matters: George Gamow Memorial Volume; Reines, F., Ed.; Colorado Associated University Press: Boulder, CO, USA, 1972; pp. $186-191$.

3. Krätze, E. Integral transformations of Bessel type. In Generalized Functions of Operational Calculus, Proc. Conf. Verna, 1975; Bulgarian Academy of Sciences: Sofia, Bulgaria, 1979; pp. 148-165.

4. Mathai, A.M. Generalized Krätzel integral and associated statistical densities. Int. J. Math. Anal. 2012, 6, 2501-2510.

5. Mathai, A.M.; Saxena, R.K.; Haubold, H.J. The H-Function: Theory and Applications; Springer: New York, NY, USA, 2010.

6. Coelho, C.A.; Arnold, B.C. Finite Form Representations for Meijer G and Fox H Functions; Lecture Notes in Statistics 223; Springer Nature Switzerland: Basel, Switzerland, 2019.

7. Mathai, A.M. Mellin convolutions, statistical distributions and fractional calculus. Fract. Calc. Appl. Anal. 2018, 21, 376-398. [CrossRef]

8. Mathai, A.M. Fractional integral operators involving many matrix variables. Linear Algebra Appl. 2014, 446, 196-215. [CrossRef]

9. Attenburger, R.; Haitz, C.; Timmer, J. Analysis of phase-resolved partial discharge patterns of voids based on a stochastic process approach, J. Phys. D Appl. Phys. 2002, 35, 1149-1163. [CrossRef]

10. Mathai, A.M. A pathway to matrix-variate gamma and normal densities. Linear Algebra Appl. 2005, 396, 317-328. [CrossRef]

11. Mathai, A.M.; Haubold, H.J. Pathway model, superstatistics, Tsallis statistics and a generalized measure of entropy. Phys. A 2007, 375, 110-122. [CrossRef]

12. Tsallis, C. Introduction to Nonextensive Statistical Mechanics: Approaching a Complex World; Springer: New York, NY, USA, 2009.

13. Mathai, A.M.; Haubold, H.J.; Tsallis, C. Pathway model and nonextensive statistical mechanics. Sun Geosph. 2015, 10, 157-162.

14. Beck, C. Superstatistics: Theoretical concepts and physical applications. In Anomalous Transport: Foundations and Applications; Klages, R., Radons, G., Sokolov, I.M., Eds.; Wiley-VCH: Weinheim, Germany, 2008; pp. 433-457.

15. Mathai, A.M.; Haubold, H.J. A versatile integral in physics and astronomy and Fox's H-function. Axioms 2019, 8, 122. [CrossRef]

16. Kobayashi, K. Plane wave diffraction by a strip: Exact and asymptotic solutions. J. Phys. Soc. Jpn. 1990, 60, 1891-1905. [CrossRef]

17. Kobayashi, K. Generalized gamma function occurring in wave scattering problem. J. Phys. Soc. Jpn. 1991, 60, 1501-1512. [CrossRef]

18. Mathai, A.M. On products and ratios of three or more generalized gamma variables. J. Indian Soc. Probab. Stat. 2016, 17, 79-94. [CrossRef]

19. Kiryakova, V.S. Multiple (multi-index) Mittag-Leffler functions and relations to generalized fractional calculus. J. Comput. Appl. Math. 2000, 118, 241-259. [CrossRef]

20. Gorenflo, R.; Kilbas, A.A.; Mainardi, F.; Rogosin, S.V. Mittag-Leffler Functions, Related Topics and Applications; Springer: New York, NY, USA, 2014.

21. Gorenflo, R.; Luchko, Y.; Mainardi, F. Analytic properties and applications of the Wright function. Fract. Calc. Appl. Anal. 1999, 2, 383-414.

22. Mainardi, F.; Luchko, Y.; Pagnini, G. The fundamental solution of the space-time fractional diffusion equations. Fract. Calc. Appl. Anal. 2001, 4, 153-192.

23. Mathai, A.M. Jacobians of Matrix Transformations and Functions of Matrix Arguments; World Scientific Publishing: New York, NY, USA, 1997.

24. Mathai, A.M.; Princy, T. Multivariate and matrix-variate Maxwell-Boltzmann and Raleigh densities. Phys. A 2017, 468, 668-676. [CrossRef]

(C) 2020 by the authors. Licensee MDPI, Basel, Switzerland. This article is an open access article distributed under the terms and conditions of the Creative Commons Attribution (CC BY) license (http:/ / creativecommons.org/licenses/by/4.0/). 